\title{
Femtosecond imaging with digital holography
}

\section{Ye $\mathrm{Pu}$, Martin Centurion, Demetri Psaltis}

Ye Pu, Martin Centurion, Demetri Psaltis, "Femtosecond imaging with digital holography," Proc. SPIE 6311, Optical Information Systems IV, 63111K (30 August 2006); doi: 10.1117/12.688468

SPIE. Event: SPIE Optics + Photonics, 2006, San Diego, California, United States 


\title{
Femtosecond Imaging with Digital Holography \\ Ye Pu*, Martin Centurion, Demetri Psaltis \\ Dept. of Electrical Engineering, California Institute of Technology, Pasadena, CA, USA 91125
}

\begin{abstract}
We describe a holographic technique capable of sampling dynamic events at 150 femtosecond time resolution. We apply the technique to the study of the nonlinear propagation of high energy pulses through gas and condensed media. The holograms are recorded as a digitized image from a CCD camera and reconstructed numerically to retrieve the refractive index change during the nonlinear optical process. We show dramatic differences in the pulse propagation characteristics depending on the strength of the nonlinear coefficient of the material and it's time response. Both positive and negative index changes have been measured in different media. The holographic technique allows us to distinguish the very fast positive index changes that are generally attributable to the Kerr nonlinearity from the negative index changes that result from free electrons generated by multiphoton ionization.
\end{abstract}

Keywords: Holography, Kerr effect, Self-focusing, Optical filamentation, Optical turbulence, Phase retrieval.

\section{INTRODUCTION}

The propagation of femtosecond optical pulses in optical Kerr media has received considerable attention in recent years. ${ }^{1-7}$ When an intense light pulse propagates in a Kerr medium, the strong electric field in the pulse induces a refractive index change in the medium by $\Delta n=n_{2} I$, where $n_{2}$ is the second-order refractive index, and $I$ is the intensity of the pulse. The optical Kerr response can originate from many mechanisms, such as electronic polarization, molecular orientation, and thermal effects, with drastically differing magnitude and response time. At femtosecond timescale, however, the Kerr response is dominated by electronic polarization.

In most materials, $n_{2}$ is positive and causes the femtosecond pulse to self-focus. ${ }^{8}$ In particular, when the peak power $P$ of the pulse is greater than the critical power $P_{c r} \approx \lambda^{2} / 8 n_{0} n_{2},{ }^{9}$ the pulse collapses into highly localized filaments. ${ }^{10}$ In unconstrained three-dimensional space, diffraction alone is unable to arrest the collapsing, ${ }^{11}$ and the beam collapses catastrophically until a higher-order nonlinear defocusing process stops it. The results of the balance between the Kerr self-focusing and the higher-order nonlinear defocusing effects range from intermittent focusing-defocusing cycles to optical solitons. When $P>>P_{c r}$, the beam breaks up into roughly $N_{f i l}=P / P_{c r}$ filaments. The regime of $P>>P_{c r}$ is particularly interesting because the additional nonlinear processes result in rich optical phenomena, such as dynamic interactions between optical solitons ${ }^{6}$ and optical turbulence. ${ }^{12}$ In order to understand the complex behavior that results at the high optical power it is important to record the induced index changes with high spatial and temporal resolution and also distinguish the sign of the index change.

Holography has long been known to be capable of capturing dynamic events. ${ }^{13-15}$ High-resolution recording of small changes in the refractive index, one of the most manifesting observables during ultrafast laser pulse propagation in a Kerr media, is only resolvable through digital holography recorded on an electronic imager. ${ }^{16}$ Although light propagation through liquids with comparable spatial resolution has been indirectly observed by media containing fluorescent dye, ${ }^{17}$ the intrinsic nanosecond-level response time of fluorescent dyes is incapable of capturing the fast dynamics of femtosecond pulses.

In this paper we describe a holographic probing technique that allows for the measurement of the index change in the nonlinear optical media during and after the femtosecond pulse propagation. The technique was first demonstrated in the measurement of laser-induced plasma in air, ${ }^{16}$ and will be reported in greater depth elsewhere. ${ }^{18}$ Femtosecond laser allows us to sample very fast dynamic events with 150 -fs temporal resolution. We demonstrate this technique in both gas and condensed media. Specifically, we have recorded holographic microscopic images for the optical filaments in air, water, and Carbon Disulfide $\left(\mathrm{CS}_{2}\right)$. Fine spatial details are revealed from these holographic reconstructions.

* Dept. of Electrical Engineering, MS 136-93, California Institute of Technology, Pasadena, CA 91125; yepu@sunoptics.caltech.edu 


\section{EXPERIMENTAL SETUP}

Figure 1 illustrates the experimental setup for the femtosecond holographic recording. We use an amplified Ti:Sapphire laser operating at $800 \mathrm{~nm}$ wavelength as the light source. The energy and duration of the laser pulse is $2 \mathrm{~mJ}$ and $150 \mathrm{fs}$ full width half maximum (FWHM), respectively. The pulse is split into a pump beam and a probe beam. A major portion of the pulse energy $(1.5 \mathrm{~mJ})$ goes into the pump beam, and the rest into the probe beam. After a variable delay, the pump beam is focused into the test section with an achromatic lens (L3) of $5 \mathrm{~cm}$ focal length. The peak power of the pump pulse is $10 \mathrm{GW}$, and a set of neutral density filters (ND) is used to adjust the pump power in the test section. The probe pulse propagates in a direction perpendicular to the pump and captures the nonlinear optical process in the test section where the pump beam is focused. The test section is imaged with a magnification of $M=f_{2} / f_{1}$ at the image plane through a 4-F system consisting of a microscope objective (L4, $\times 100.25 \mathrm{NA}$ ) and an achromatic lens (L5). We record in-line (Gabor) hologram ${ }^{19}$ with the 14-bit digital CCD camera at a distance $L$ from the image plane. The hologram is then numerically reconstructed to retrieve the phase of the probe beam passing the test section.

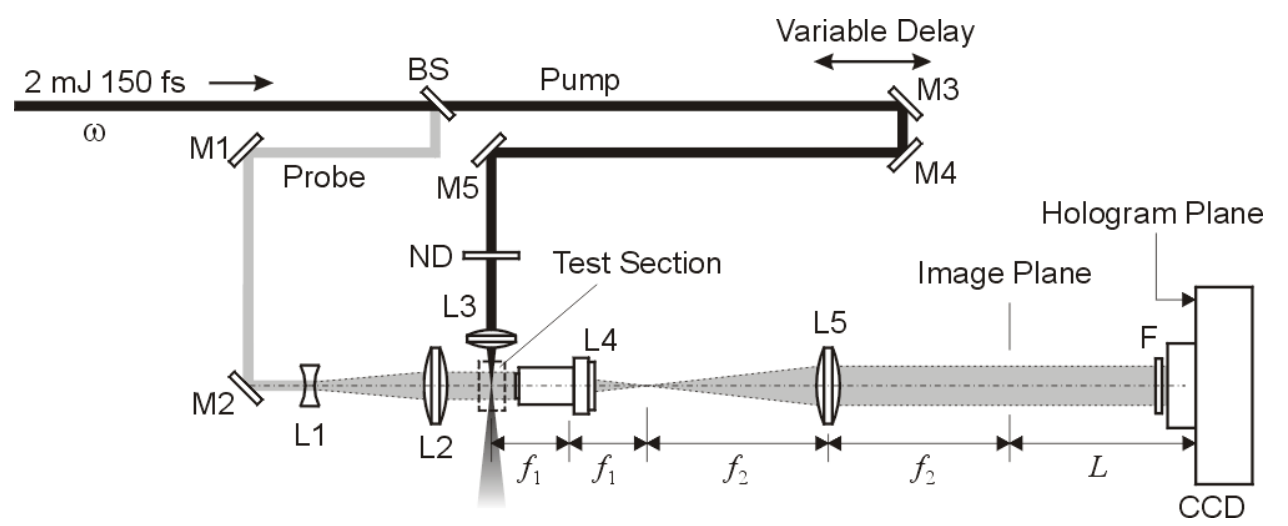

Figure 1. Experimental setup for femtosecond holographic recording of optical Kerr effect and the associated plasma filaments due to self-focusing. An intense femtosecond laser pulse is split into a pump and a probe pulse. The pump pulse is focused by L3 and undergoes self-focusing due to optical Kerr effect, forming optical filaments. A probe pulse transverses the test section during and after the pump pulse goes through, forming an in-line hologram on the CCD sensor. The system has a magnification of $f_{2} / f_{1}$. L1 - L3, L5: Lenses. L4: microscope objective. M1 - M5: Mirrors. BS: Beamsplitter. ND: Neutral density filter.

\section{DIGITAL HOLOGRAPHY FOR PHASE RECOVERY}

The inline hologram, as described above, records the interference pattern between the unperturbed probe wave and the wave diffracted from the object under investigation. The object refers to the spatial structure of the refractive index change $\Delta n$ induced by the nonlinear optical process (optical filamentation). Figure 2 shows an example digital hologram captured by the CCD camera. The spatial resolution of the hologram is $2184 \times 1472$ pixels, and the pixel size is $6.8 \mu \mathrm{m} \times 6.8 \mu \mathrm{m}$. The hologram is captured with $\mathrm{L}=30 \mathrm{~cm}$ and $\mathrm{M}=12.5$. To remove spatial defects in the probe beam profile, a separate image frame of the probe beam alone is subtracted from the hologram.

We numerically back propagate this hologram to reconstruct the object wave front and retrieve the phase change $\Delta \phi$ due to the object. The reconstruction algorithm calculates the convolution between the holographic fringes and a Fresnel phase kernel. ${ }^{20}$ It is in general difficult to calculate the corresponding index change if $\Delta n$ contains complex threedimensional structures. Here we assume the filament structure is simple and ignore the diffraction through the thin filament. Hence the phase change $\Delta \phi$ that the probe pulse undergoes after passing through the object is calculated as

$$
\Delta \phi(x, y)=\int_{0}^{s} 2 \pi \Delta n(x, y, z) / \lambda d z,
$$

where $s$ is the size of the plasma filament. 


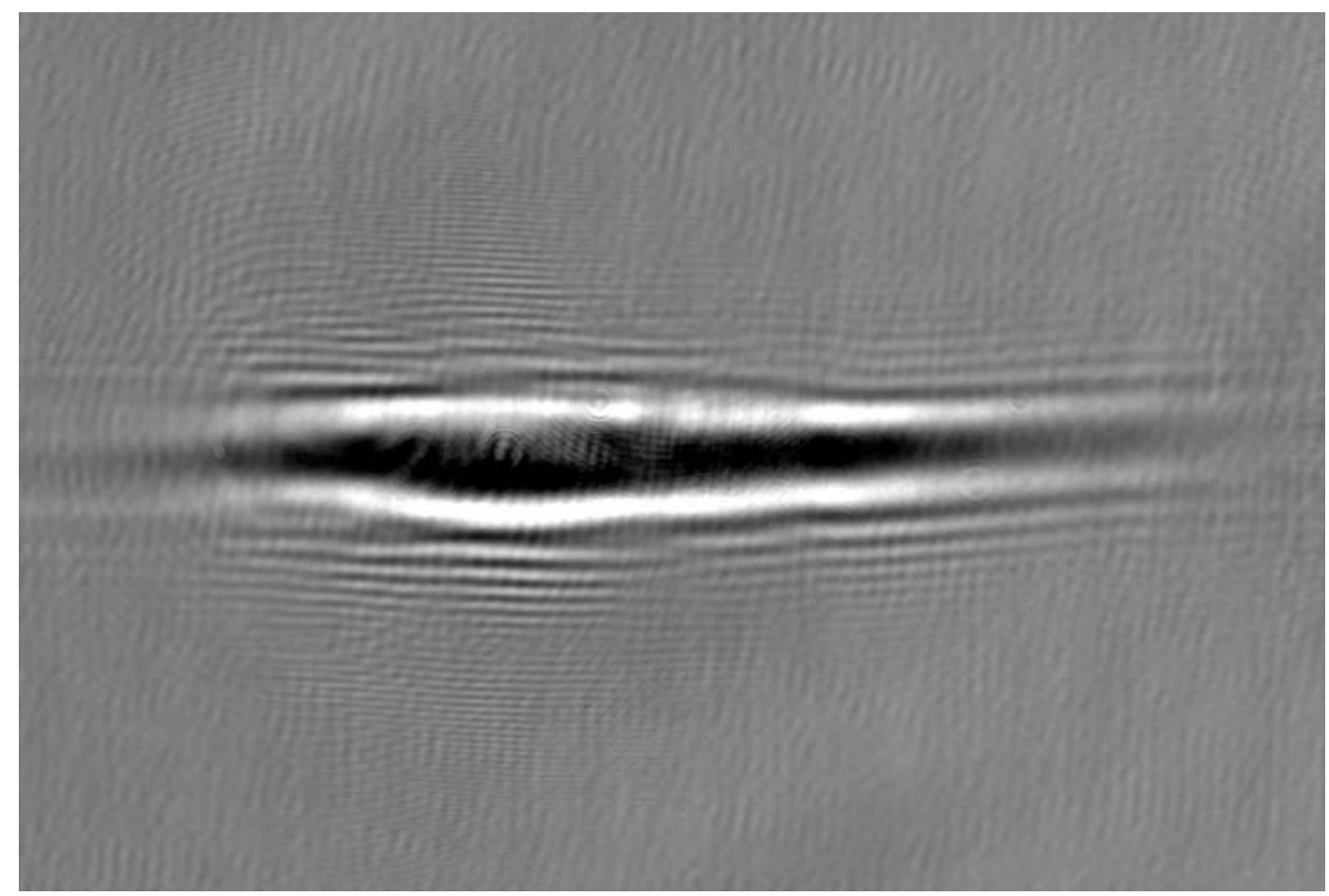

Figure 2. Digital in-line hologram of the plasma filaments in air. The hologram, having a resolution of $2184 \times 1472$ pixels and a pixel size of $6.8 \mu \mathrm{m} \times 6.8 \mu \mathrm{m}$, is captured with $\mathrm{L}=30 \mathrm{~cm}$ and $\mathrm{M}=12.5$ at a temporal moment approximately 1 ps after the pump pulse has passed. A separate image frame of the probe beam alone is subtracted from the hologram to remove spatial defects in the probe beam profile.

The phase of the object wave recovered through in-line holography is subject to system errors. Figure 3 (a) shows the reconstructed phase from a simulated hologram of two one-dimensional Gaussian phase object at $\lambda=800 \mathrm{~nm}$ and $L=30 \mathrm{~cm}$. The waist size of the objects in the object space (before magnification) is $100 \mu \mathrm{m}$ and $200 \mu \mathrm{m}$, and the peak phase change of both objects is -1 radian. Compared to the original phase distribution, the recovered phase is typically $6 \%$ smaller for the $100 \mu \mathrm{m}$ object, and close to $50 \%$ for a $200 \mu \mathrm{m}$ object. The recovered phase of smaller objects is more accurate than that of larger objects. In addition, the recovery of phase information from a single hologram is based on the assumption that the object is a pure phase object that does not absorb nor scatter light. Since all real objects involve light absorption and scattering, further measurement error in the recovered phase is inevitable. In our experiments, on the other hand, the overall lateral beam size of the pump in the test section does not exceed $100 \mu \mathrm{m}$, and the fine structures of self-focusing filaments induced by the pump are smaller than $15 \mu \mathrm{m}$ in all cases. Furthermore, the objects in these experiments are all pure phase objects with very small refractive index change, and the error due to light scattering is of little significance. Therefore, we estimate the relative measurement error in the retrieved phase is below $6 \%$.

The reconstructed phase also suffers inherent artifacts outside the object region due to the in-line superposed virtual image. In general, in-line holography is unable to retrieve the phase for large, strongly diffracting objects, since it becomes very difficult to separate the real image from the virtual image. Figure 3 (b) shows an example phase distribution obtained from the hologram shown in Figure 2. Since only the phase within the extent of the object is of physical significance, we mark the region outside the object as invalid to rule out the influence of the artifacts in the reconstruction. In the following sections, we only present the recovered phase in the valid region. 


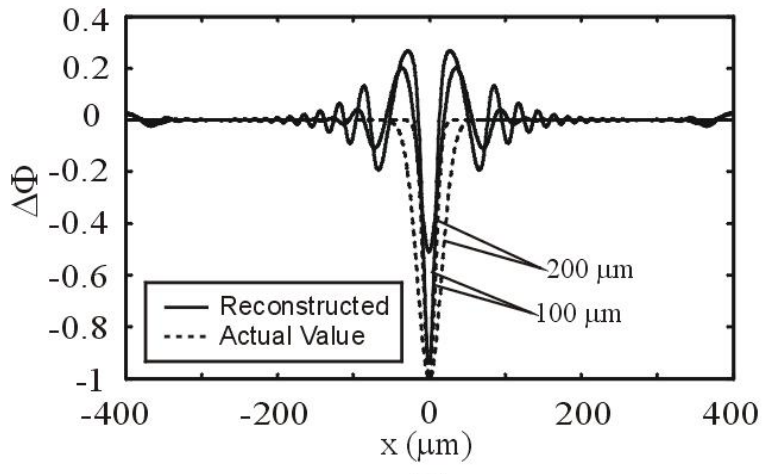

(a)

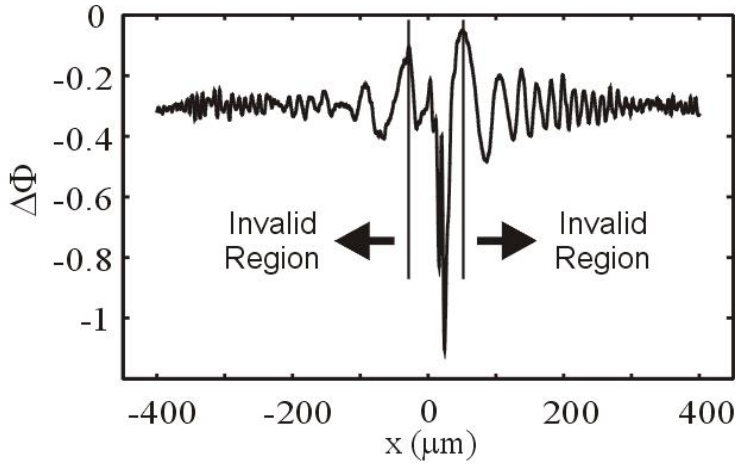

(b)

Figure 3. Error estimation for the phase recovery from in-line holography. (a) Phase recovery from a simulated hologram by a $100 \mu \mathrm{m}$ and a $200 \mu \mathrm{m}$ linear phase object. The relative phase error is around $6 \%$ for a $100 \mu \mathrm{m}$ object, and close to $50 \%$ for a $200 \mu \mathrm{m}$ object. (b) Phase reconstruction from the hologram shown in Figure 2. The phase fluctuation in the invalid region as marked does not have physical meanings.

\section{EXPERIMENTAL RESULTS}

We have performed digital holographic measurements of the optically-induced refractive index changes in air, water, and $\mathrm{CS}_{2}$ with great spatial details. The underlying nonlinear mechanisms in gases and condensed media are very different, leading to vastly distinct physical phenomena of self-organizing light structures.

\subsection{Optical Filamentation in Air}

Self-channeling of femtosecond pulses in air has been previously studied, ${ }^{4}$ which involves intermittent focusing and defocusing cycles. When the input beam collapses into filaments, free electrons are generated at the filament core as a result of typically eight- to ten-photon-ionization of air. The free electrons negatively contribute to the refractive index and cause the filament to defocus. The dynamic competition between the self-focusing and defocusing effects results in long range optical filaments. Here we capture one cycle of the focusing and defocusing process with a focused femtosecond laser pulse with microscopic spatial resolution. Given the nonlinear refractive index of $n_{2}=5.0 \times 10^{-19}$ $\mathrm{cm}^{2} / \mathrm{W}$, direct visualization of the Kerr response in air is difficult. Nonetheless, significant refractive index change is resulted from the free electrons in the plasma filament trailing the optical pulse, which reveals the propagation history of the optical filaments. The air media in the test section is pumped with full power, which corresponds to approximately six times of the critical power of $1.8 \mathrm{GW}$ for self-focusing in air.

The map of phase change $\Delta \phi$ in the probe beam after passing the plasma filament is shown in Figure 4 (a), which is captured at a temporal moment approximately $1 \mathrm{ps}$ after the pump pulse has passed. This change of index as calculated from Equation (1) is a direct measure of the free electron density (i.e. the plasma density) $N_{e}$ as ${ }^{9}$

$$
n_{0}+\Delta n=\varepsilon^{1 / 2}=\left(1-4 \pi N_{e} q^{2} / m \omega^{2}\right)^{1 / 2},
$$

where $n_{0} \approx 1$ is the refractive index of air, $\varepsilon$ is the electrical permittivity, $q$ is the coulomb charge of electron, $m$ is the mass of electron, and $\omega$ is the frequency of the probe pulse. From this relationship we obtain the density of free electron as $N_{e}=\left(-2 \Delta n+\Delta n^{2}\right) N_{c r}$, where $N_{c r}=m \omega^{2} / 4 \pi q^{2}=1.7 \times 10^{21} \mathrm{~cm}^{-3}$ is the critical plasma density at $800 \mathrm{~nm}$ wavelength. We show the spatial distribution of $N_{e}$ in Figure 4 (b). The smallest resolvable feature in the filament structures is approximately $4 \mu \mathrm{m}$, which is very close to the diffraction limit of the holographic system. Assuming the overall size of the plasma filament $s=60 \mu \mathrm{m}$, we estimate the maximum index change is approximately $-2.12 \times 10^{-3}$, corresponding to a free electron density of $7.03 \times 10^{18} \mathrm{~cm}^{-3}$. 

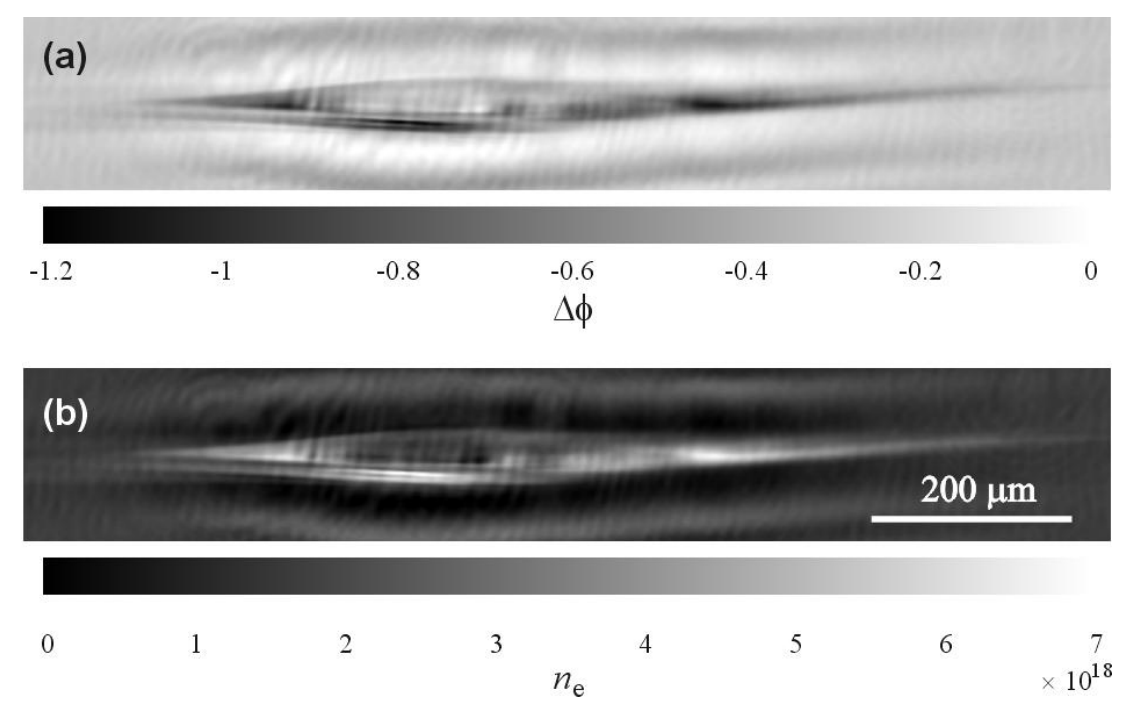

Figure 4. (a) Phase reconstruction from the hologram of the plasma filament in air. (b) Map of free electron density calculated from the phase change. The propagation is from the left to the right. Filament breakup and recombination is clearly shown.

A slightly modified configuration of the holographic technique is also capable of capturing time sequences of the plasma-generation process. To achieve this, we replace mirror M2 in Figure 1 with a mirror array consisting of four mirror segments and generate four probe pulses at distinct, carefully tuned angle and time. The four pulses spatially overlap in the test section, albeit temporally separated. In order to ensure the events are sampled at approximately the same angle the separation angle between the probe pulses has to be sufficiently small (a few degrees), which limits the effective angular aperture of each individual hologram. The CCD camera records the four spatially separated holograms with magnification $M=2.5$ at a distance $L=16 \mathrm{~cm}$. The map of free electron density calculated from the four holograms is shown in Figure 5, where the first probe pulse is set at the onset of the plasma, and the temporal separation between the pulses roughly $1 \mathrm{ps}$. The energy of the pump laser pulse is the same as in the previous experiment. Despite the reduced spatial resolution as a result of the limited angular aperture, this result gives a dynamic picture of the time evolution of the plasma density in the optical filaments in air.
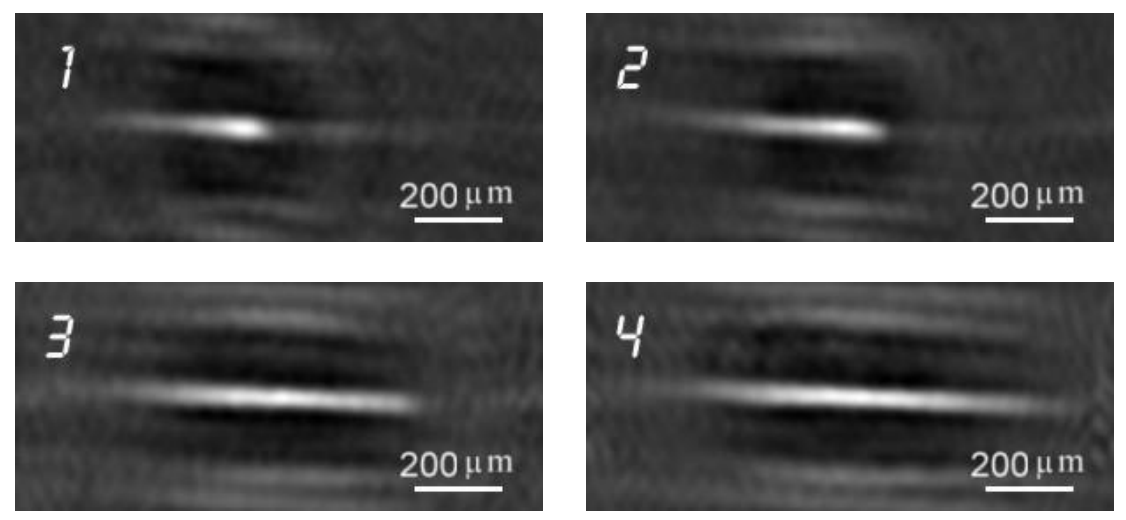

Figure 5. Temporal evolution of the electron density in the plasma filaments induced by a single femtosecond optical pulse.

The relative time of the snapshots $1-4$ is $0,1,2,2.7 \mathrm{ps}$, respectively. Due to the small angular aperture limited by the probe separation angle, fine structures in the filamentation process are not recovered.

\subsection{Optical Filamentation in Water}

We investigate the filamentation of a focused femtosecond laser pulse in water with the holographic technique described in Section 2. Nonlinear propagation and optical breakdown of femtosecond laser pulses in water has been the subject of recent investigations. ${ }^{2,5,21}$ Simulations ${ }^{21}$ show that, at femtosecond timescale, the ionization in aqueous media 
is purely due to multiphoton ionization, and the electron density can reach $10^{18} \mathrm{~cm}^{-3}$ at the focal point. Different from the similar shadowgraph approach employed in elsewhere, ${ }^{2}$ our technique is able to resolve the phase change in the plasma filament quantitatively with high spatial resolution. Our results show some interesting deviations form previous theoretical studies.

The nonlinear refractive index of water is $n_{2}=4.1 \times 10^{-16} \mathrm{~cm}^{2} / \mathrm{W}$, which is approximately 800 times that of air. The corresponding critical power for self-focusing is $P_{c r}=1.47 \mathrm{MW}$. Although the phase change due to the optical Kerr effect is too weak to directly visualize through the phase reconstruction, the plasma trail that the optical filaments generates is clearly resolvable through holography.

Figure 6 shows the maps of phase reconstructed from three independent holograms for the plasma filaments following a focused $1 \mathrm{~mJ}$ femtosecond pulses in deionized water. At an input power of $P \approx 4.5 \times 10^{3} P_{c r}$, the optical beam breaks up into a large number of filaments (approximately 4-5 $\mu \mathrm{m}$ in diameter) long before reaching the focal point. Optical turbulence is clearly presented, manifested by completely different spatial filament structures each pulse. Due to the extremely complex three-dimensional structures of the filaments, the spatial distribution of the refractive index change $\Delta n$ and the free electron density $N_{e}$ cannot be recovered reliably from the phase change $\Delta \phi$. Nonetheless, the amplitude of $\Delta \phi$ qualitatively reflects the density of the free electrons. Figure 6 suggests that $\Delta \phi$ and thus $N_{e}$ reaches its peak more than $0.5 \mathrm{ps}$ after the pump pulse has passed. The evolution of the phase change cannot be the result of thermal effect due to the energy transfer during recombination, which is a much slower process. Therefore, additional ionization after the passage of the pulse is evident, and multiphoton ionization does not dominate the density of free electrons. This "postponed response" could be a result of a complex interactions between many processes, and further investigation is required to understand this phenomenon.
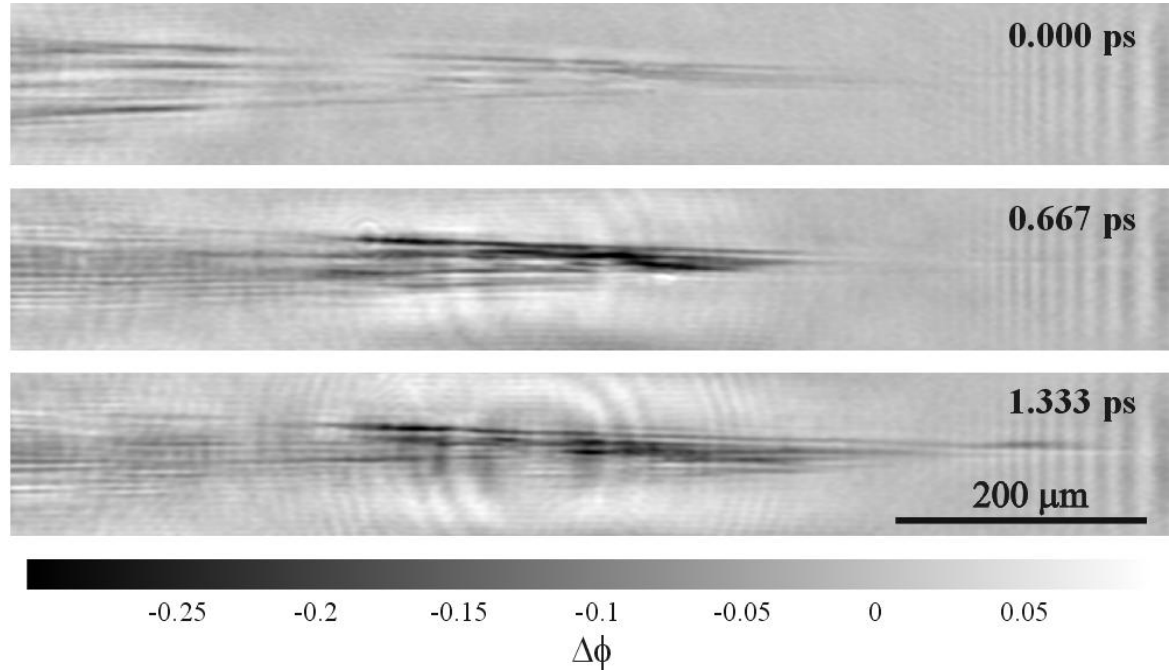

Figure 6. Phase reconstruction of the plasma filaments in the wake of a focused $1 \mathrm{~mJ}$ femtosecond laser pulse in water. The phase maps are obtained from three independent holograms at time delays as shown. The propagation is from the left to the right. The much longer response time compared to the pump pulse width indicates that the contribution from avalanche ionization following the initial multiphoton ionization dominates the density of free electrons in the plasma filaments.

\subsection{Optical Filamentation in $\mathrm{CS}_{2}$}

Self-focusing of intense laser light was first observed in $\mathrm{CS}_{2},{ }^{8}$ an excellent nonlinear optical material, in 1966 with a Qswitched Ruby laser. The origin of the wide accepted nonlinear refractive index of $n_{2}=3.2 \times 10^{-14} \mathrm{~cm}^{2} / \mathrm{W}$ in $\mathrm{CS}_{2}$ is molecular orientation, with a characteristic response of 2 ps. At femtosecond timescale, however, the molecular response vanishes, and the nonlinear response of $\mathrm{CS}_{2}$ is dominated by electronic polarization. A value of $n_{2}=3 \times 10^{-15}$ has been measured previously using femtosecond pulses, ${ }^{7}$ which corresponds to a critical power of $P_{c r}=165 \mathrm{~kW}$ for 
self focusing. The reduced nonlinear refractive index of $\mathrm{CS}_{2}$, on the other hand, is still sufficient to enable the direct recovery of the phase change due to the optical Kerr effect.

Figure 7 shows the phase map of a focused femtosecond pulse at pulse energy of 51,13,3 $\mu \mathrm{J}$, corresponding to an input power of 2000, 500, 125 times of $P_{c r}$. The instantaneous nature of the electronic optical Kerr response is clearly shown.

Due to the molecular response time of $2 \mathrm{ps}$ in $\mathrm{CS}_{2}$, a trace of the filaments trailing the femtosecond pulse is faintly observable. The filament structures change drastically from shot to shot, indicating the existence of optical turbulence. The input beam split into many filaments roughly $10 \mu \mathrm{m}$ each, which then travel a long distance without further collapsing. Instead of the focusing-defocus cycle observed in air and water, the filaments in CS2 actually behave like optical solitons. At $3 \mu \mathrm{J}$ input energy the number of filaments is reasonably low, and we are able to estimate the refractive index change in each filament is approximately $\Delta n=3.1 \times 10^{-4}$. Taking the measured filament size ${ }^{22}$ of $12 \mu \mathrm{m}$ and the calculated $P_{c r}$, we calculate the nonlinear refractive index of $\mathrm{CS}_{2}$ as $n_{2}=\Delta n A / P_{c r}=2.5 \times 10^{-15} \mathrm{~cm}^{2} / \mathrm{W}$, where $A$ is the area of the filament. This value is in good agreement with the measurement with other approach. At any power level, plasma (indicated by negative refractive index change) is clearly absent in $\mathrm{CS}_{2}$. This is in contrast to the consensus in the literature that self-guiding of the optical filaments occurs as a consequence of dynamic balance between Kerr self-focusing and defocusing effects in the electron plasma generated through the multiphoton ionization. Numerical simulations ${ }^{6}$ have shown that a negative fifth-order nonlinearity, i.e. $n_{4}<0$, could be very possible to stop the collapsing of the filaments in $\mathrm{CS}_{2}$.
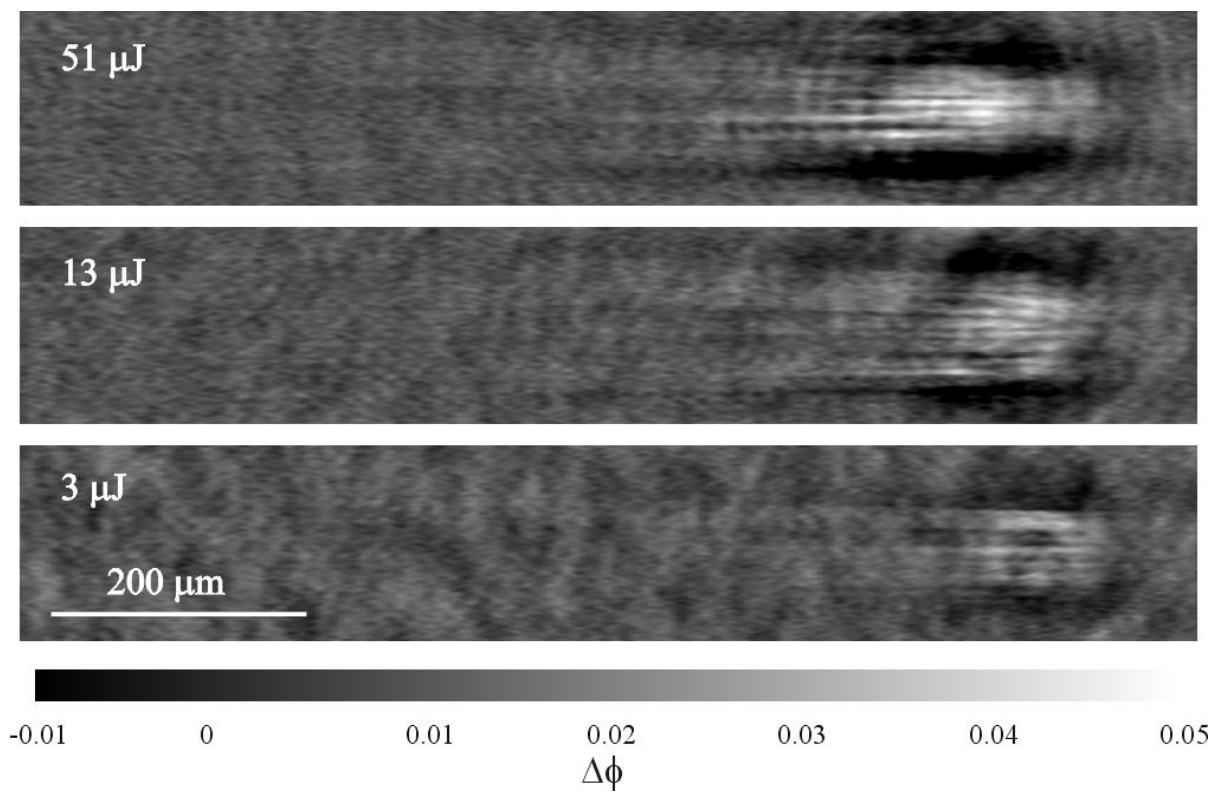

Figure 7. Phase reconstruction of a focused femtosecond laser pulse in $\mathrm{CS}_{2}$ with pulse energy of approximately 51,13 , and $3 \mu \mathrm{J}$. The propagation is from the left to the right. Because $\mathrm{CS}_{2}$ possesses a large $\mathrm{n}_{2}$, the positive phase change as a result of the optical Kerr effect is directly visible. The absence of plasma in the filament wakes (manifested by negative phase change) suggests that a negative fifth-order nonlinear process other than multiphoton ionization could be responsible for preventing the optical filaments from collapsing. Due to the molecular response time of 2 ps in $\mathrm{CS}_{2}$, a trace of the filaments trailing the femtosecond pulse is faintly observable.

\section{CONCLUSION}

We have described a holographic method of measuring the spatial map of phase change in nonlinear optical processes with rich spatial detail. We demonstrate this technique in both gas (air) and condensed media (water and $\mathrm{CS}_{2}$ ) in the regime where the input power is many times greater than the self-focusing critical power. The phase map obtained in air allows us for the measurement free electrons generated at the trail of a femtosecond pulse propagating in air. This technique allows us to capture a single hologram with fine spatial resolution (4 microns). We also obtained a time- 
sequence of multiple holograms with reduced spatial resolution in a single-shot experiment, revealing the dynamic evolution of the plasma in air. In aqueous media, holographic measurements show that the intense femtosecond pulse split into numerous filaments, which leave plasma trails behind them. The temporal evolution of the plasma suggests that multiphoton ionization could not be the dominating process, as predicted in theory, in the generation of free electrons. In $\mathrm{CS}_{2}$, the measurements reveal an absence of plasma, and the optical filaments forms solitons traveling a long distance without further collapsing. This signifies the existence of a negative fifths order nonlinear response that is responsible for the stopping of the collapsing.

\section{ACKNOWLEDGEMENTS}

The authors wish to thank Zhiwen Liu and Theodor W. Hänsch for helpful discussions. This research was supported by the Defense Advanced Research Projects Agency Center for Optofluidic Integration. Funding for M. Centurion was provided through the Center for the Physics of Information as part of the Caltech Information Science and Technology initiative.

\section{REFERENCES}

1. A. Brodeur, F. A. Ilkov, and S. L. Chin, "Beam filamentation and the white light continuum divergence", Opt. Commun. 129, 193-198 (1996).

2. E. Abraham, K. Minoshima, and H. Matsumoto, "Femtosecond laser-induced breakdown in water: timeresolved shadow imaging and two-color interferometric imaging", Opt. Commun. 176, 441-452 (2000).

3. A. Couairon, S. Tzortzakis, L. Berge, M. Franco, B. Prade, and A. Mysyrowicz, "Infrared femtosecond light filaments in air: simulations and experiments", J. Opt. Soc. Am. B-Opt. Phys. 19, 1117-1131 (2002).

4. A. Braun, G. Korn, X. Liu, D. Du, J. Squier, and G. Mourou, "Self-Channeling of High-Peak-Power Femtosecond Laser-Pulses in Air", Opt. Lett. 20, 73-75 (1995).

5. A. Dubietis, G. Tamosauskas, I. Diomin, and A. Varanavicius, "Self-guided propagation of femtosecond light pulses in water", Opt. Lett. 28, 1269-1271 (2003).

6. M. Centurion, Y. Pu, and D. Psaltis, "Self-organization of spatial solitons", Opt. Express 13, 6202-6211 (2005).

7. R. A. Ganeev, A. I. Ryasnyansky, M. Baba, M. Suzuki, N. Ishizawa, M. Turu, S. Sakakibara, and H. Kuroda, "Nonlinear refraction in CS2", Appl. Phys. B-Lasers Opt. 78, 433-438 (2004).

8. E. Garmire, R. Y. Chiao, and C. H. Townes, "Dynamics and Characteristics of Self-Trapping of Intense Light Beams", Phys. Rev. Lett. 16, 347-\& (1966).

9. R. W. Boyd, Nonlinear Optics (Academic Press, Boston, MA, 2002).

10. $\quad$ Y. Silberberg, "Collapse of Optical Pulses", Opt. Lett. 15, 1282-1284 (1990).

11. P. L. Kelley, "Self-Focusing of Optical Beams", Phys. Rev. Lett. 15, 1005-\& (1965).

12. M. Mlejnek, M. Kolesik, J. V. Moloney, and E. M. Wright, "Optically turbulent femtosecond light guide in air", Phys. Rev. Lett. 83, 2938-2941 (1999).

13. N. Abramson, "Light-in-Flight Recording - High-Speed Holographic Motion-Pictures of Ultrafast Phenomena", Appl. Optics 22, 215-232 (1983).

14. H. T. Hsieh, D. Psaltis, O. Beyer, D. Maxein, C. von Korff Schmising, K. Buse, and B. Sturman, "Femtosecond holography in lithium niobate crystals", Opt. Lett. 30, 2233-2235 (2005).

15. Z. W. Liu, M. Centurion, G. Panotopoulos, J. Hong, and D. Psaltis, "Holographic recording of fast events on a CCD camera", Opt. Lett. 27, 22-24 (2002).

16. M. Centurion, Y. Pu, Z. W. Liu, D. Psaltis, and T. W. Hansch, "Holographic recording of laser-induced plasma", Opt. Lett. 29, 772-774 (2004).

17. H. Schroeder and S. L. Chin, "Visualization of the evolution of multiple filaments in methanol", Opt. Commun. 234, 399-406 (2004).

18. M. Centurion, Y. Pu, and D. Psaltis, "Holographic Capture of Femtosecond Pulse Propagation", Submitted to J. Appl. Phys. (2006).

19. D. Gabor, "A New Microscopic Principle", Nature 161, 777-778 (1948). 
20. H. J. Kreuzer, M. J. Jericho, I. A. Meinertzhagen, and W. B. Xu, "Digital in-line holography with photons and electrons", J. Phys.-Condes. Matter 13, 10729-10741 (2001).

21. P. K. Kennedy, "A First-Order Model for Computation of Laser-Induced Breakdown Thresholds in Ocular and Aqueous-Media .1. Theory", IEEE J. Quantum Electron. 31, 2241-2249 (1995).

22. M. Centurion, Y. Pu, M. K. Tsang, and D. Psaltis, "Dynamics of filament formation in a Kerr medium", Phys. Rev. A 71, (2005). 